\title{
Protective Carbon Deposition for Superior FIB Prepared (S)TEM Specimens
}

\author{
Brandon Van Leer, Young-Chung Wang and Lucille A. Giannuzzi
}

FEI Company, 5350 NE Dawson Creek Drive, Hillsboro, OR 97124

Focused ion beam (FIB) assisted chemical vapor deposited (CVD) metal layers such as Pt or W are routinely used to planarize specimens to avoid curtaining artifacts during FIB cross-sectioning for SEM or TEM specimen preparation [1]. Using a DualBeam ${ }^{\mathrm{TM}}$ (FIB/SEM) instrument, electron beam assisted deposition (EBAD) layers are often deposited as an alternative to ion beam assisted deposited (IBAD) layers to prevent ion implantation damage to the outer most $\sim 50 \mathrm{~nm}$ region of the sample [2]. Since the EBAD process takes $\sim 20 \mathrm{x}$ longer than the IBAD process, thick (i.e., $>\sim 200$ $\mathrm{nm})$ EBAD layers are seldom used for the entire protective layer. Thus, most protective layers consist of a deposit of $\sim 50-200 \mathrm{~nm}$ thick EBAD layer followed by an IBAD layer.

The Pt-based GIS precursor is an organometallic-based material, and therefore, neither the IBAD nor the EBAD Pt is pure metal, and nominally consists of a heterogeneous mixture of nanocrystalline $(\mathrm{Pt}+\mathrm{C})$ grains [ref. 3 and see FIG. 1]. Excess oxygen may also be observed in the deposit, and of course, Ga is observed in the IBAD coating [3]. A Carbon GIS source is also available for DualBeam deposition use. A protective $\mathrm{C}$ layer may be preferred for specimen preparation over the higher atomic number Pt or W, particularly for Z-contrast high angled annular dark field (HAADF) STEM imaging since the low atomic number $\mathrm{C}$ layer will not dominate the contrast during analysis. Over the past few years, low energy $\mathrm{Ga}^{+}$ion FIB techniques using the DualBeam (FIB/SEM) have been exploited to prepare specimens that are capable of achieving the information limit (i.e., sub-angstrom) of aberration corrected (S)TEM instruments [4,5]. Below, we compare the use of EBAD and IBAD Pt versus $\mathrm{C}$ deposition protective layers for high quality (S)TEM specimens.

EBAD/IBAD C and EBAD/IBAD Pt were deposited on Si and FIB prepared for STEM analysis. Each face of the specimen was FIB milled using $\mathrm{Ga}^{+}$ions at $30 \mathrm{keV}$ and 88.5 degrees incident angle, followed by $5 \mathrm{keV}$ at 85 degrees incident angle, then $2 \mathrm{keV}$ polishing at 82 degrees incidence angle. FIG. 1 shows $30 \mathrm{keV}$ bright field (BF) STEM images of (left) Pt deposited on Si and (right) C deposited on Si. FIG. 2 shows $30 \mathrm{keV}$ dark field (DF) STEM images of (left) Pt deposited on Si and (right) $\mathrm{C}$ deposited on $\mathrm{Si}$. Note that the grain size of the EBAD Pt is 1-3 nm and the IBAD Pt is 10$20 \mathrm{~nm}$. The larger grained IBAD Pt compared to the EBAD Pt is consistent with findings in [3]. In comparison, the EBAD and IBAD $C$ layers yield sub-nanometer grains, with the IBAD $\mathrm{C}$ grains slightly larger than the EBAD C grains (see FIG. 2).

TRIM calculations [6] show that C sputters faster than Pt by $\sim 24 \%$ at the defined conditions at 30 $\mathrm{keV}$, but the difference in sputter yield between $\mathrm{C}$ and Pt increases to $>30 \%$ at $5 \mathrm{keV}$. Thus, during the low energy milling steps, the slower milling Pt grains sets up edge effects which manifests as curtaining artifacts observed in the specimen shown in FIG 1 and FIG 2. However, the Si samples protected with the smaller and more homogenous grain sized $\mathrm{C}$ deposition layer yield no observable curtaining artifacts in the Si protected by $\mathrm{C}$ deposition. Thus, the use of $\mathrm{C}$ deposition as a protective layer yields less curtaining FIB milling artifacts than Pt and is a better alternative for high resolution (S)TEM specimen preparation techniques. 


\section{References}

[1] Introduction to Focused Ion Beams, eds. L.A. Giannuzzi and F.A. Stevie, Springer (2005).

[2] B.W. Kempshall et al., J. Vac. Sci. Tech. B, 20(1) (2002) 286.

[3] R.M. Langford et al., Microelectronic Engineering, 84 (2007) 784.

[4] Lucille A. Giannuzzi et al., Microsc. Microanal. 11(Suppl 2), (2005), 828.

[5] C Kisielowski et al., Microsc. Microanal. 14 (2008) 469.

[6] J.F. Ziegler et al., www.srim.com, (2008).
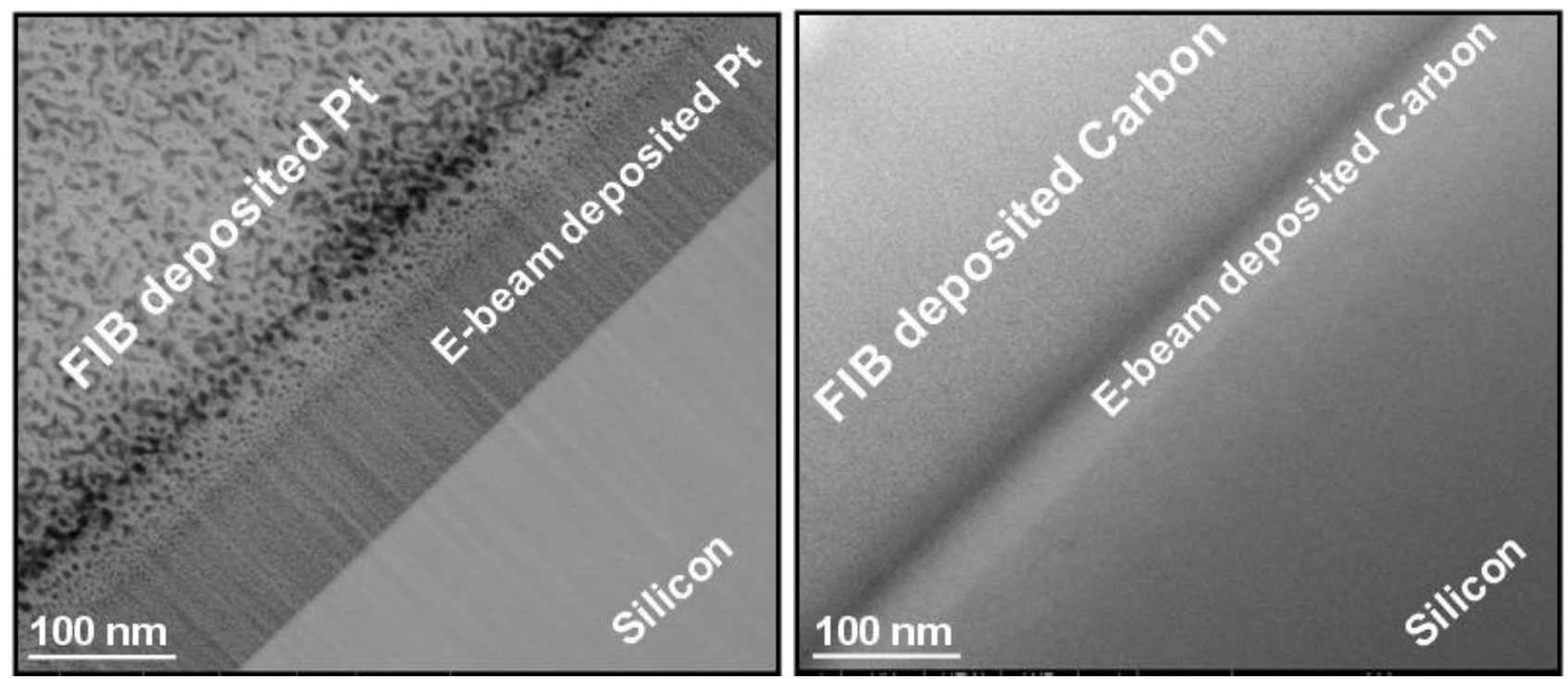

FIG.1. $30 \mathrm{keV}$ bright field STEM images of FIB prepared specimens. The left image shows Pt on Si and the right image shows $\mathrm{C}$ deposition on $\mathrm{Si}$.
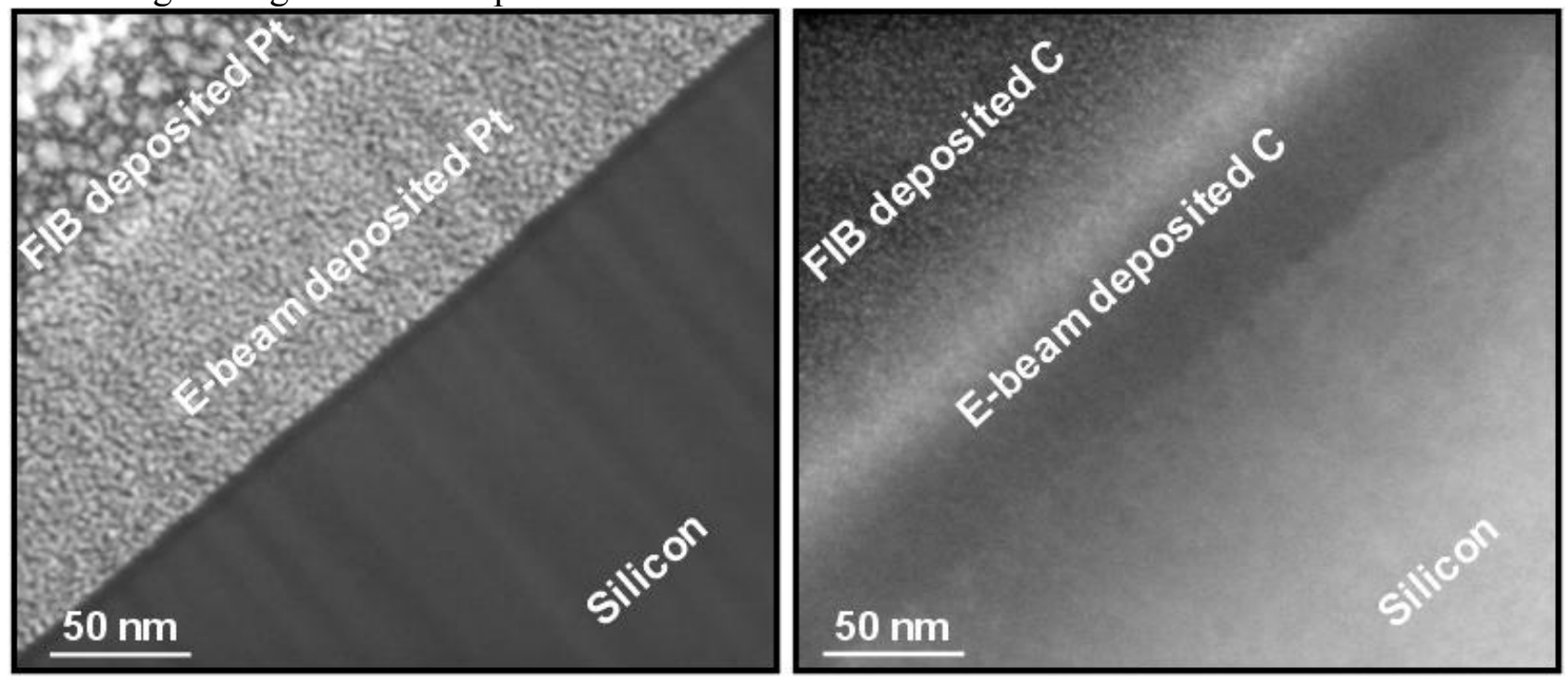

FIG.2. $30 \mathrm{keV}$ dark field STEM images of FIB prepared specimens. The left image shows Pt on $\mathrm{Si}$ and the right image shows $\mathrm{C}$ deposition on $\mathrm{Si}$. 